\title{
Erratum to: Magnetic Properties of Dysprosium Doped Cobalt Ferrite Nanoparticles Synthesized by Starch-Assisted Sol-Gel Auto-combustion Method
}

\author{
Raghvendra Singh Yadav ${ }^{1}$. Jaromir Havlica ${ }^{1}$ Ivo Kuřitka ${ }^{2}$ Zuzana Kozakova ${ }^{2}$. \\ Martin Palou ${ }^{1}$ - Eva Bartoníčková1 ${ }^{1}$ Martin Boháć ${ }^{1}$ - Františka Frajkorová ${ }^{1}$. \\ Jiri Masilko ${ }^{1}$ - Lukas Kalina ${ }^{1}$ • Miroslava Hajdúchová ${ }^{1}$ - Vojtěch Enev ${ }^{1}$. \\ Jaromir Wasserbauer ${ }^{1}$
}

Published online: 9 December 2015

(c) Springer Science+Business Media New York 2015

Erratum to: J Supercond Nov Magn (2015)

$$
\text { 28:2097-2107 }
$$

10.1007/s10948-015-3009-6

In the original paper, the authors want to include one funding agency in the acknowledgement section. The corrected acknowledgement is presented in this Erratum:

Acknowledgments This work was supported by Project Excellent Teams (CZ.1.07/2.3.00/30.0005), CZ.1.07/2.3.00/30.0039 and Sustainability and Development, REG LO1211, National Programme for Sustainability I (Ministry of Education, Youth and Sports) at the Materials Research Centre, Brno University of Technology, and Project Centre of Polymer Systems (Reg. Number: CZ.1.05/2.1.00/03.0111) at Tomas Bata University in Zlín, Czech Republic.

The authors would like to apologize for any inconvenience caused.

The online version of the original article can be found at http://dx.doi.org/10.1007/s10948-015-3009-6.

Raghvendra Singh Yadav

yadav@fch.vutbr.cz

1 Materials Research Centre, Brno University of Technology,

Purkyňova 464/118, 61200 Brno, Czech Republic

2 Centre of Polymer Systems, University Institute, Tomas Bata University in Zlín, Nad Ovčírnou 3685, 76001 Zlín, Czech Republic 